\title{
Stiff, strong, and tough hydrogels with good chemical stability
}

\section{Citation}

$\mathrm{Li}$, Jianyu, Zhigang Suo, and Joost J. Vlassak. 2014. "Stiff, Strong, and Tough Hydrogels with Good Chemical Stability." J. Mater. Chem. B 2 (39): 6708-6713. doi:10.1039/c4tb01194e.

\section{Published Version}

doi:10.1039/C4TB01194E

\section{Permanent link}

http://nrs.harvard.edu/urn-3:HUL.InstRepos:13919158

\section{Terms of Use}

This article was downloaded from Harvard University's DASH repository, and is made available under the terms and conditions applicable to Open Access Policy Articles, as set forth at http:// nrs.harvard.edu/urn-3:HUL.InstRepos:dash.current.terms-of-use\#OAP

\section{Share Your Story}

The Harvard community has made this article openly available.

Please share how this access benefits you. Submit a story.

Accessibility 


\title{
Stiff, strong, and tough hydrogels with good chemical stability
}

\author{
Jianyu Li, Zhigang Suo and Joost J. Vlassak* \\ School of Engineering and Applied Sciences, Harvard University, Cambridge, MA 02138, USA \\ E-mail: vlassak@seas.harvard.edu
}

\begin{abstract}
Most hydrogels have poor mechanical properties, severely limiting their scope of applications. Here we report on a hybrid hydrogel that combines extremely high stiffness, strength, and toughness, while maintaining physical integrity in electrolyte solutions. The hydrogel consists of a hydrophilic polymer network that is covalently cross-linked and a second polymer network that can crystallize. We show that the crystallites serve as physical cross-links for the second network. The crystallites contribute to the high stiffness and strength of the hydrogel; they unzip and dissipate energy under deformation, and reform due to the incompatibility of the two polymers in the hydrogel. The crystallite-toughened hydrogel can achieve an elastic modulus of $5 \mathrm{MPa}$, a strength of $2.5 \mathrm{MPa}$, and a fracture energy of $14,000 \mathrm{Jm}^{-2}$. Unlike alginate-based hybrid hydrogels, this hydrogel preserves its mechanical properties in electrolyte solutions and could be considered for further development in a variety of engineering applications.
\end{abstract}


Recent developments in the area of hydrogels promise to greatly expand their scope of applications. ${ }^{1-7}$ Many applications require hydrogels to endure significant mechanical loads in aggressive environments. Examples range from biomedical applications such as artificial cartilage in tissue engineering, ${ }^{8}$ to engineering applications such as swellable packers in the oil industry, ${ }^{9}$ or artificial nerves and muscles in the nascent field of soft machines. ${ }^{10}$ Substitutes for cartilage require high stiffness $(1 \mathrm{MPa})$, high toughness $\left(1,000 \mathrm{Jm}^{-2}\right)$, and high water content $(60$ $80 \%) .{ }^{11}$ Materials for oil packers require high stiffness, high strength, and chemical stability; they need to resist deformation, sustain sealing pressure (up to $34 \mathrm{MPa}$ ) and be stable in saline water. ${ }^{9}$ Materials for artificial nerves and muscles require high resistance to mechanical damage,${ }^{12}$ and tolerance of concentrated electrolyte for ionic conductance. ${ }^{10}$ Most hydrogels have low stiffness $(\sim 10 \mathrm{kPa})$, strength $(\sim 100 \mathrm{kPa})$ and toughness $\left(\sim 10 \mathrm{Jm}^{-2}\right) ;^{13}$ and they often degrade in electrolyte solutions. ${ }^{14}$ There is a strong need for mechanically robust hydrogels with good chemical stability.

Despite recent progress, developing hydrogels that are both mechanically robust and chemically stable is still a challenge. ${ }^{13,15}$ Breaking covalent bonds in tough double network gels results in permanent and irreversible damage to the network. ${ }^{16}$ Formation of hydrophobic associations is limited by low solubility of the hydrophobes ${ }^{17}$ Hydrogen bonds have very low association strength in hydrogels due to competition of water for binding sites. ${ }^{18}$ Ionic cross-links are particularly effective in toughening hydrogels - alginate-polyacrylamide hydrogels in which the alginate is cross-linked with calcium ions exhibit remarkably high toughness. ${ }^{3,19}$ The ionic cross-link is, however, vulnerable to mobile ions, which are often encountered under physiological and engineering conditions, ${ }^{8,10}$ and the performance of ionically cross-linked hydrogels degrades markedly in electrolyte solutions. ${ }^{2,14}$ 
As an alternative to ionic cross-links, the use of crystallites to cross-link a network has great potential to overcome these limitations: crystallites serve as physical cross-links that are reversible and stable when exposed to mobile ions. Polyvinyl alcohol (PVA) is an example of a polymer that can form crystallites. PVA is widely used and has seen extensive development for biomedical applications. $^{20,21}$ In 1975, Peppas discovered that a sequence of freeze and thaw cycles could produce PVA hydrogels where crystallites serve as cross-links. ${ }^{20}$ Unfortunately, hydrogels synthesized using this method are compliant and brittle. ${ }^{22}$ It is possible to achieve higher stiffness and toughness using a dry-anneal method, but only at the expense of a much lower water content (Supplementary Fig. S1). ${ }^{23}$ Muratoglu and coworkers polymerized acrylamide monomers in the pores of a PVA hydrogel to form uncross-linked chains, and showed that the equilibrium water content of the resulting gels increased with acrylamide content, while the coefficient of friction, tear strength and creep resistance decreased. ${ }^{21}$

Here we propose that a hybrid network of a crystalline polymer and a covalently crosslinked hydrophilic polymer may form a hydrogel with robust mechanical properties and good chemical stability: the crystalline polymer can generate a large number of crystallites to serve as physical cross-links that are both stable and reversible; the covalently cross-linked hydrophilic polymer maintain the elasticity of the network during deformation and controls the swelling of the hydrogel. We describe one such hybrid hydrogel that combines extremely high stiffness, strength, and toughness. The hydrogel consists of a hydrophilic polyacrylamide (PAAm) polymer network that is covalently cross-linked and a PVA network that forms crystallites. We show that the PVA crystallites result in a high cross-link density, thus producing a gel of remarkable stiffness and strength. The crystallites unzip under deformation, dissipating energy in the process and yielding a hydrogel with exceptional toughness. After deformation, unzipped crystallites recover at room temperature due to the incompatibility of the two polymers in the gel. The 
crystallite-toughened hydrogel can achieve an elastic modulus of $5 \mathrm{MPa}$, a strength of $2.5 \mathrm{MPa}$, and a fracture energy of $14,000 \mathrm{Jm}^{-2}$. Moreover, these properties are stable, even in concentrated electrolyte solutions.

\section{Results}

We prepared the hybrid hydrogels in a simple three-step protocol. First, we form a crosslinked network of PAAm in the presence of PVA chains. Unlike the gel synthesized by Muratoglu et al., the PAAm chains are covalently cross-linked to increase the retraction force of the network and to prevent leakage of the polymer chains. ${ }^{24}$ Second, we dry and anneal the gel at elevated temperature. The drying process removes water and concentrates the PVA in the mixture. The annealing process enhances the mobility of PVA chains and promotes crystallization. $^{21,23}$ This step generates a much higher density of PVA crystallites than the freezethaw method developed by Peppas. ${ }^{20}$ The resulting dry gel is translucent, indicative of phase separation (Supplementary Fig. S2); evidently PAAm and PVA are not mutually soluble. ${ }^{25}$ X-ray diffraction measurements confirmed the formation of crystallites (Supplementary Fig. S3). In the final step, we re-hydrate the gel by immersing it in water for a period of time. The gel absorbs water and swells until it reaches equilibrium. Fourier transform infrared spectroscopy confirmed that the PVA-PAAm hydrogel is essentially a superposition of PVA and PAAm hydrogels (Supplementary Fig. S4).

Toughening effect of crystallites. To evaluate the mechanical behavior of the hybrid gels, we performed tensile experiments on two types of hydrogels: an entangled PVA-PAAm hydrogel and a crystallized PVA-PAAm hydrogel. The former was not subject to the dry-anneal step and the PVA chains in this gel were simply entangled in the cross-linked polyacrylamide network; the 
latter went through the standard three-step protocol. Samples of both gels were stretched to rupture (Fig. 2a). The nominal stress is the loading force divided by the cross-sectional area of the undeformed sample. The stretch ratio is the current length divided by the initial length of undeformed sample. The entangled PVA-PAAm hydrogel is compliant and soft. In contrast, the crystallized PVA-PAAm hydrogel is stiff and strong, evidently the result of a high density of physical cross-links. Each sample was stretched to a stretch ratio of 2.5 and then unloaded to its initial length (Fig. 2b). In the entangled PVA-PAAm hydrogel, the absence of crystallites results in relatively little hysteresis, and the sample behaves more or less elastically. In the crystallized PVA-PAAm hydrogel, on the other hand, the crystallites unzip and dissipate a significant amount of energy. This dissipated energy manifests as significant hysteresis in the stress-stretch curve, indicative of a very tough gel. This behavior is similar to that of pure PVA, for which the evolution of crystallites under deformation has been characterized with small-angle $\mathrm{X}$ ray scattering and is well documented in the literature. ${ }^{26}$ The unzipping of crystallites was further confirmed by the anisotropic swelling of gels after uniaxial deformation and the resulting anisotropic microstructure as revealed by confocal microscopy (Supplementary Fig. S5): the crystallites unzip preferentially in the deformation direction, allowing the gel to further expand in this direction on swelling.

Stability in electrolyte solutions. We performed tensile experiments on the PVA-PAAm gel and an alginate-PAAm, before and after soaking overnight in a saline solution $\left(9 \mathrm{gL}^{-1}\right)$. The results are shown in Fig. 2c. The alginate-PAAm hydrogel is a tough gel formed by covalently and ionically cross-linked polymers. The electrostatic interactions between alginate chains and calcium ions form ionic cross-links that unzip and dissipate energy under deformation. ${ }^{3}$ The sodium ions in the saline solution compete with the calcium ions for the binding sites on the 
alginate chains, resulting in an unzipping of the ionic cross-links. After soaking, the strength of the alginate-PAAm gel is significantly reduced and the gel has lost its energy dissipation mechanism - the stress-stretch curve no longer shows any hysteresis. By contrast, soaking in saline has no effect on the stress-stretch curves of the PVA-PAAm gels, even in highly concentrated electrolyte solutions (Supplementary Fig. S6). Unlike ionic cross-link, the crystallites in PVA do not rely on electrostatic interaction and remain intact in electrolytes with a high content of monovalent ions. Furthermore, unlike alginate, the PVA network is not susceptible to hydrolysis: the mechanical properties of the PVA-based gel do not degrade after storing in distilled water for as long as three months (Fig. 2d).

Recovery of crystallite-toughened hydrogels. We performed compression tests on cylindrical samples with a diameter of $5 \mathrm{~mm}$ to investigate the recovery properties of these hydrogels. Each sample was first compressed to a stretch ratio of 0.2 and then unloaded. The sample was then stored at room temperature for various durations of time and subjected to another compression test (Fig. 2e). We quantify the ability of the crystallites to reform by the recovery ratio, i.e., the work performed during the second compression test normalized by the work performed during the first test. The recovery ratio increases to more than $90 \%$ in less than 24 hours (Fig. 2 f). The phenomenon is understood as follows. As the crystallites unzip under deformation, the covalently cross-linked network provides the force to re-store the original shape of the gel. Since PAAm and PVA are incompatible, there is a driving force for phase separation; the PVA chains aggregate and reform the crystallites at room temperature. ${ }^{27}$ This process is relatively slow because of the large molecular weight of the PVA, but the rate of recovery increases with increasing covalent cross-link density (Supplementary Fig. S7). A similar phenomenon is also observed in entangled 
PVA-PAAm hydrogel, which is initially transparent, but slowly turns translucent after storing at room temperature (Supplementary Fig. S8).

Mechanical properties of crystallite-toughened hydrogels. To evaluate the effect of acrylamide on the properties of the dry-annealed PVA-PAAm gel, we prepared a series of hydrogels with various ratios of acrylamide to (acrylamide plus polyvinyl alcohol) and performed tensile tests. The results are summarized in Fig. 3. As the acrylamide content increases, both the strength (Fig. 3a) and stiffness (Fig. 3b) of the hydrogel decreases. The fracture energy was measured by performing tensile tests on notched samples using a geometry known as the pure shear test. ${ }^{3,28}$ Similar to the other mechanical properties, the fracture energy of the hydrogels decreases with increasing acrylamide content (Fig. 3c). This trend is inversely correlated with the equilibrium water content (Fig. 3d): as the acrylamide content of a gel increases, so does its affinity for water, and the gel absorbs more water. ${ }^{29}$ In the limit of no acrylamide, the gel has very good mechanical properties, but its water content is low. At $25 \%$ acrylamide, the hydrogel has a relatively high water content of $62 \%$, while maintaining a good combination of mechanical properties: an elastic modulus of $5 \mathrm{MPa}$, a strength of $2.5 \mathrm{MPa}$ and a fracture energy of 14,000 $\mathrm{Jm}^{-2}$. The mechanical behavior of these hybrid gels is well described by the rule of mixtures applied to the PVA and PAAm phases (Supplementary Fig. S9), and varies with the covalent cross-link density of the PAAm component (Supplementary Fig. S10). The properties of PVA/PAAm hybrid gels can be tailored over a broad range. The swelling behavior of the hydrogel can be tuned by controlling the hydrophilicity of the covalent network. For instance, sodium acrylate can be copolymerized with acrylamide to provide a large ionic osmotic pressure and so increase the equilibrium water content of the gel (Supplementary Fig. S11). 
In Figure 4, we present two Ashby charts for a variety of soft materials. In terms of strength and stiffness, PVA-PAAm hydrogels outperform most hydrogels, including alginatepolyacrylamide hydrogels and polyampholyte hydrogels (Fig. 4a). Traditional PVA hydrogels prepared using freeze/thaw cycles are strong, but compliant and brittle with a toughness of only $500 \mathrm{Jm}^{-2} .{ }^{22}$ Bacterial cellulose-gelatin hydrogels have high strength, but are brittle and rupture at small strains. ${ }^{30}$ With respect to stiffness and fracture energy, PVA-PAAm hydrogels do better than all other hydrogels (Fig. 4b). Considering that these gels contain $60-80 \%$ water, achieving such high strength and fracture resistance is quite remarkable.

Our results suggest that hydrogels with excellent mechanical performance and chemical stability can be prepared by combining hydrophilic and crystalline polymers. With appropriate processing, the crystalline polymer can form a high density of crystallites that serve as cross-links resulting in high stiffness, strength, and toughness; the network formed by the hydrophilic polymer maintains the shape of the hydrogel, provides a strong driving force to reform crystallites, and increases the equilibrium water content. The hydrogels remain stable in physiological solutions. This strategy has a large pool of material candidates. Beside polyvinyl

alcohol, a variety of other crystalline polymers can be used, including polyurethane, ${ }^{31}$ poly-lactic acid and polyethylene glycol. ${ }^{32}$ The combination of high stiffness, high strength, fracture resistance and recoverability along with chemical stability, biocompatibility, facile synthesis, and low cost make these materials ideal candidates for load-bearing applications

\section{Method}

Hydrogel synthesis. The polyvinyl alcohol-polyacrylamide hydrogel was synthesized using the following protocol. Polyvinyl alcohol powder (PVA, Mw 89000-98000, hydrolysis >99\%) was dissolved in distilled water at $90{ }^{\circ} \mathrm{C}$; the mixture was stirred overnight until the solution became 
homogeneous. The PVA solution was then mixed with acrylamide (AAm), N,N'methylenebisacrylamide (MBAA), tetramethyl-ethylenediamine (TEMED) and ammonium persulfate (APS) in this sequence. The weights of TEMED and APS were fixed at 0.0055 and 0.006 times that of AAm, while the weight ratio of PVA versus AAm was varied. The mixture was placed in a glass mold $\left(75 \times 45 \times 3 \mathrm{~mm}^{3}\right)$, and kept at room temperature overnight to complete the reaction.

Crystallization and rehydration. After synthesis, the hydrogels were dried at room temperature. The dry gels were subsequently heated in a vacuum furnace (Symphony, VWR). The temperature was kept at $80^{\circ} \mathrm{C}$ for 3 hours and then increased to $120^{\circ} \mathrm{C}$ for 1 hour. The gel was then fully rehydrated in distilled water, which took approximately three days. The mass of the gel was measured before and after rehydration $\left(\mathrm{m}_{\text {dry }}, \mathrm{m}_{\text {wet }}\right)$ using an analytical balance. The equilibrium water content was calculated using $1-m_{d r y} / m_{\text {wet }}$.

Tensile tests. A rectangular strip of gel $\left(75 \times 45 \times 3 \mathrm{~mm}^{3}\right)$ was glued to two rigid acrylate clamps $\left(75 \times 20 \times 1.5 \mathrm{~mm}^{3}\right)$. Both unnotched and notched samples were prepared for tensile tests. For notched samples, an edge crack of length $35 \mathrm{~mm}$ was cut using a razor blade in the middle of the gauge section of the sample. An Instron machine (model 3342 with a $1000 \mathrm{~N}$ load cell) was used. The stretch rate was fixed at $2 \mathrm{~min}^{-1}$. The signals of force and extension were recorded throughout the experiment.

Compression tests. Uniaxial compression tests were performed on gel cylinders of $5 \mathrm{~mm}$ diameters using the Instron machine. The upper platen approached the sample slowly until a rise in force was detected, indicating contact between the platen and the gel. Upon contact, the displacement rate of the platen was increased to $1 \mathrm{~mm} / \mathrm{min}$ until a pre-determined stretch ratio was achieved. The entire loading process was finished in less than five minutes to avoid any 
redistribution of solvent inside the gel, and thus maintain incompressibility. Both force and displacement were recorded continuously throughout the experiment.

\section{Acknowledgements}

The work was supported by the MRSEC (DMR-0820484) and the Kavli Institute for Bionano Science and Technology at Harvard University. It was performed in part at the Center for Nanoscale Systems at Harvard University. The authors thank David A. Weitz for providing the rheometer and confocal microscopy, and David J. Mooney for providing the Instron machine.

\section{Author contributions}

J. Y. L., Z. G. S. and J. J. V. conceived and designed the study. Z. G. S. and J. J. V. supervised

the research. J. Y. L. performed the experiments. J. Y. L., Z. G. S. and J. J. V. wrote the manuscript and all authors commented on the manuscript.

\section{Additional information}

Supplementary Information accompanies this paper at http://www.nature.com/

naturecommunications

Competing financial interests: The authors declare no competing financial interests. 


\section{References}

1 Gong, J. P., Katsuyama, Y., Kurokawa, T. \& Osada, Y. Double-network hydrogels with extremely high mechanical strength. Adv. Mater. 15, 1155-1158 (2003).

2 Sun, T. L. et al. Physical hydrogels composed of polyampholytes demonstrate high toughness and viscoelasticity. Nat. Mater. 12, 932-937 (2013).

3 Sun, J. Y. et al. Highly stretchable and tough hydrogels. Nature 489, 133-136 (2012).

4 Haraguchi, K. \& Takehisa, T. Nanocomposite hydrogels: A unique organic-inorganic network structure with extraordinary mechanical, optical, and swelling/de-swelling properties. Adv. Mater. 14, 1120-1124 (2002).

5 Bakarich, S. E., Beirne, S., Wallace, G. G. \& Spinks, G. M. Extrusion printing of ioniccovalent entanglement hydrogels with high toughness. J. Mater. Chem. B 1, 4939-4946 (2013).

6 Henderson, K. J., Zhou, T. C., Otim, K. J. \& Shull, K. R. Ionically cross-linked triblock copolymer hydrogels with high strength. Macromolecules 43, 6193-6201 (2010).

7 Sakai, T. et al. Design and fabrication of a high-strength hydrogel with ideally homogeneous network structure from tetrahedron-like macromonomers. Macromolecules 41, 5379-5384 (2008).

8 Huey, D. J., Hu, J. C. \& Athanasiou, K. A. Unlike bone, cartilage regeneration remains elusive. Science 338, 917-921 (2012).

9 Kleverlaan, M., van Noort, R. H. \& Jones, I. in SPE/IADC Drilling Conference, Society of Petroleum Engineers, Houston, Texas (2005).

10 Keplinger, C. et al. Stretchable, transparent, ionic conductors. Science 341, 984-987 (2013). 
11 ChinPurcell, M. V. \& Lewis, J. L. Fracture of articular cartilage. J. Biomech. Eng. 118, 545-556 (1996).

12 Martinez, R. V., Glavan, A. C., Keplinger, C., Oyetibo, A. I. \& Whitesides, G. M. Soft actuators and robots that Are resistant to mechanical damage. Adv. Funct. Mater 24, 3003-3010 (2014).

13 Calvert, P. Hydrogels for soft machines. Adv. Mater. 21, 743-756 (2009).

14 Kamata, H., Akagi, Y., Kayasuga-Kariya, Y., Chung, U.-I. \& Sakai, T. "Nonswellable" hydrogel without mechanical hysteresis. Science 343, 873-875 (2014).

15 Zhao, X. Multi-scale multi-mechanism design of tough hydrogels: building dissipation into stretchy networks. Soft Matter 10, 672-687 (2014).

16 Gong, J. P. Why are double network hydrogels so tough? Soft Matter 6, 2583-2590 (2010).

17 Tuncaboylu, D. C., Sari, M., Oppermann, W. \& Okay, O. Tough and self-healing hydrogels formed via hydrophobic interactions. Macromolecules 44, 4997-5005 (2011).

18 Appel, E. A., del Barrio, J., Loh, X. J. \& Scherman, O. A. Supramolecular polymeric hydrogels. Chem. Soc. Rev. 41, 6195-6214 (2012).

19 Li, J., Illeperuma, W. R., Suo, Z. \& Vlassak, J. J. Hybrid hydrogels with extremely high stiffness and toughness. ACS Macro Lett. 3, 520-523 (2014).

20 Hassan, C. M. \& Peppas, N. A. in Biopolymers PVA Hydrogels, Anionic Polymerisation Nanocomposites, Springer Berlin Heidelberg, 37-65 (2000).

21 Bodugoz-Senturk, H., Macias, C. E., Kung, J. H. \& Muratoglu, O. K. Poly (vinyl alcohol)-acrylamide hydrogels as load-bearing cartilage substitute. Biomaterials 30, 589596 (2009). 
22 Zhang, L., Zhao, J., Zhu, J. T., He, C. C. \& Wang, H. L. Anisotropic tough poly(vinyl alcohol) hydrogels. Soft Matter 8, 10439-10447 (2012).

23 Cha, W. I., Hyon, S. H., Oka, M. \& Ikada, Y. Macromol. Symp. 109, 115-126 (1996).

24 Bodugoz-Senturk, H. et al. The effect of polyethylene glycol on the stability of pores in polyvinyl alcohol hydrogels during annealing. Biomaterials 29, 141-149 (2008).

25 Mishra, S., Bajpai, R., Katare, R. \& Bajpai, A. On the mechanical strength of biocompatible semi-IPNs of polyvinyl alcohol and polyacrylamide. Microsystem Technologies 14, 193-198 (2008).

26 Miyazaki, T., Hoshiko, A., Akasaka, M., Shintani, T. \& Sakurai, S. SAXS studies on structural changes in a poly (vinyl alcohol) film during uniaxial stretching in water. Macromolecules 39, $2921-2929$ (2006).

27 Holloway, J. L., Lowman, A. M. \& Palmese, G. R. The role of crystallization and phase separation in the formation of physically cross-linked PVA hydrogels. Soft Matter 9, 826833 (2013).

28 Lake, G. J. \& Thomas, A. G. Strength of highly elastic materials. Proc. R. Soc. London, Ser. A 300, 108-119 (1967).

29 Li, J. Y., Hu, Y. H., Vlassak, J. J. \& Suo, Z. G. Experimental determination of equations of state for ideal elastomeric gels. Soft Matter 8, 8121-8128 (2012).

30 Nakayama, A. et al. High mechanical strength double-network hydrogel with bacterial cellulose. Adv. Funct. Mater. 14, 1124-1128 (2004).

31 Wu, J., Ge, Q. \& Mather, P. T. PEG- POSS multiblock polyurethanes: synthesis, characterization, and hydrogel formation. Macromolecules 43, 7637-7649 (2010).

32 Gaharwar, A. K. et al. Highly extensible bio-nanocomposite fibers. Macromol. Rapid Comm. 32, 50-57 (2011). 
33 Haque, M. A., Kurokawa, T., Kamita, G. \& Gong, J. P. Lamellar bilayers as reversible sacrificial bonds to toughen hydrogel: hysteresis, self-recovery, fatigue resistance, and crack blunting. Macromolecules 44, 8916-8924 (2011).

34 Zhang, L. et al. High strength graphene oxide/polyvinyl alcohol composite hydrogels. $J$. Mater. Chem. 21, 10399-10406 (2011).

35 Wegst, U. G. K. \& Ashby, M. F. The mechanical efficiency of natural materials. Philos. Mag. 84, 2167-2181 (2004). 


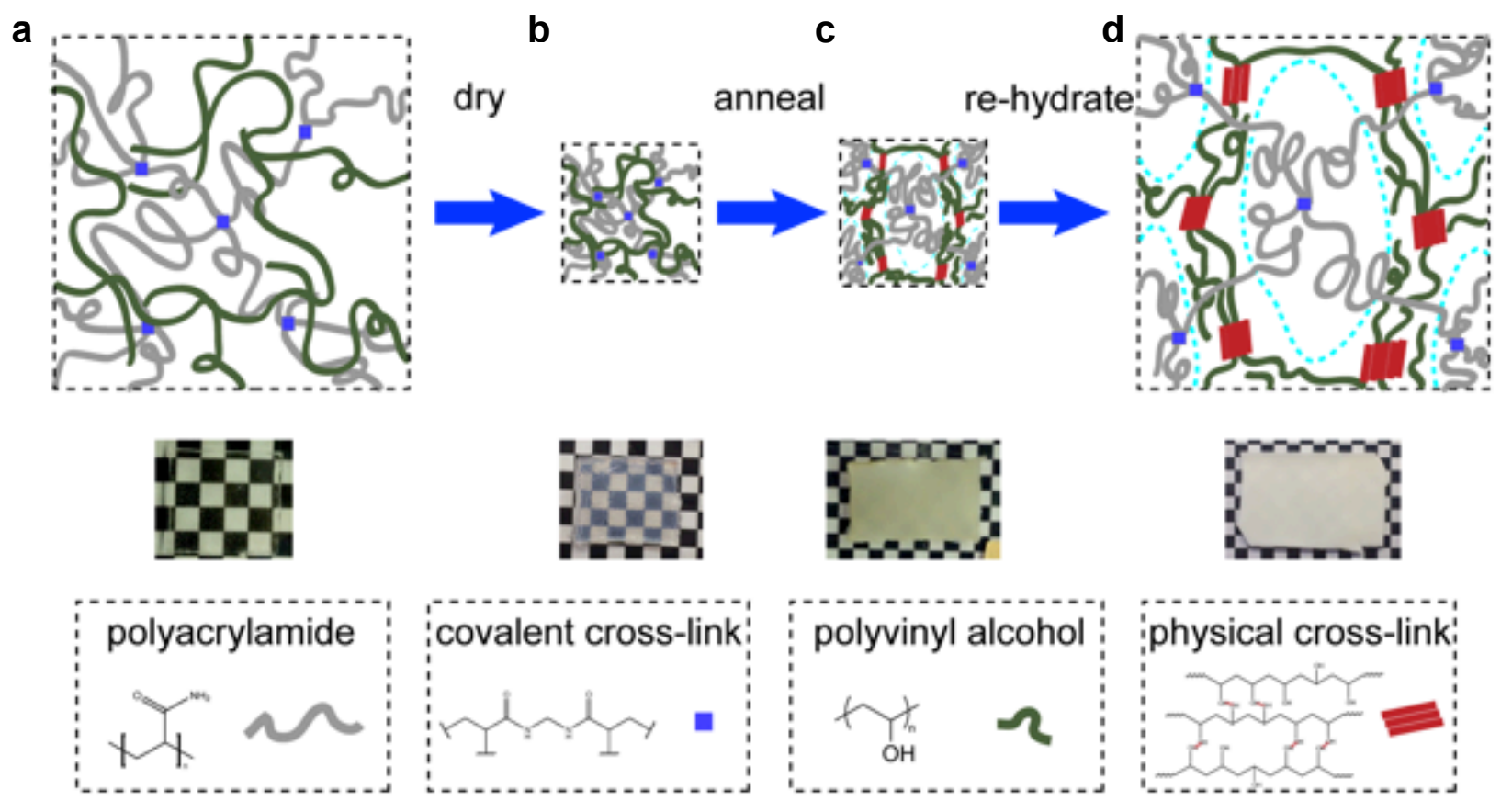

Figure 1. Synthesis and structure of the hybrid hydrogel. a, Acrylamide forms a covalently cross-linked network (PAAm, gray lines) with N,N'-methylenebisacrylate (MBAA, blue squares). The gel is dried at room temperature (b), and then annealed at elevated temperature (c). Phase separation occurs (dashed cyan lines). Polyvinyl alcohol (PVA, green lines) forms a physically cross-linked network with crystallites (red blocks). d, On rehydration in distilled water, the crystallized PVA-PAAm gel forms a hydrogel. Optical photographs of the gels were placed beneath the corresponding schematics. 


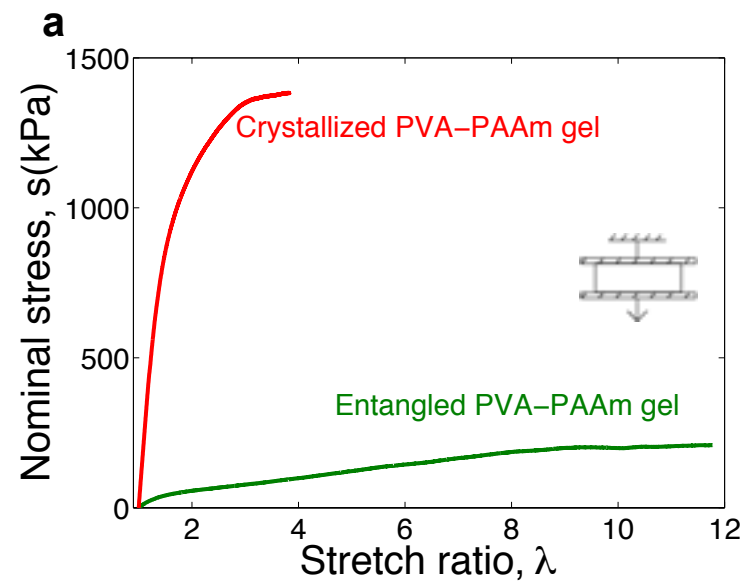

C

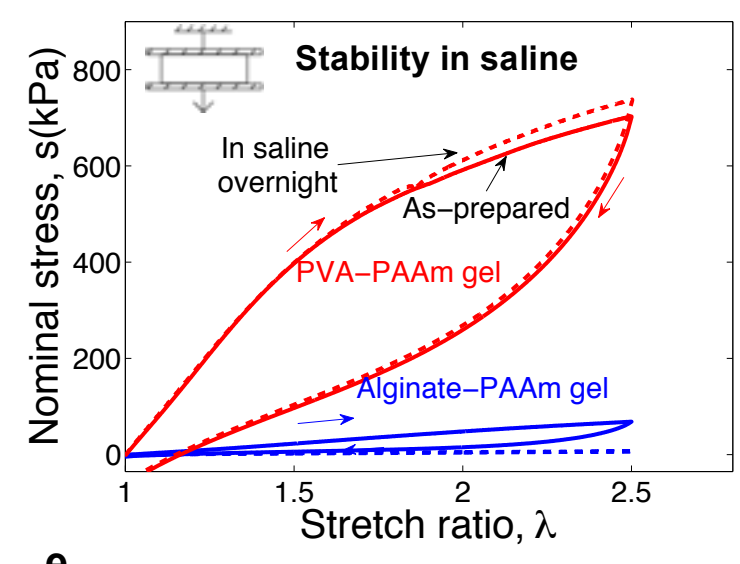

e

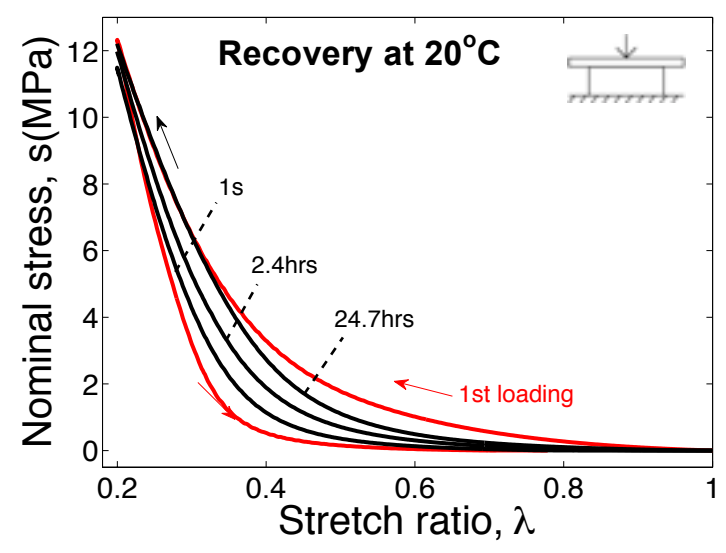

b

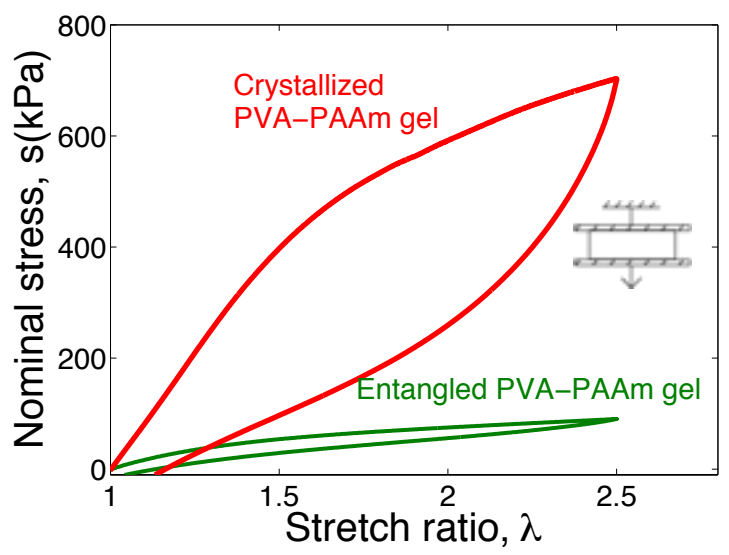

d

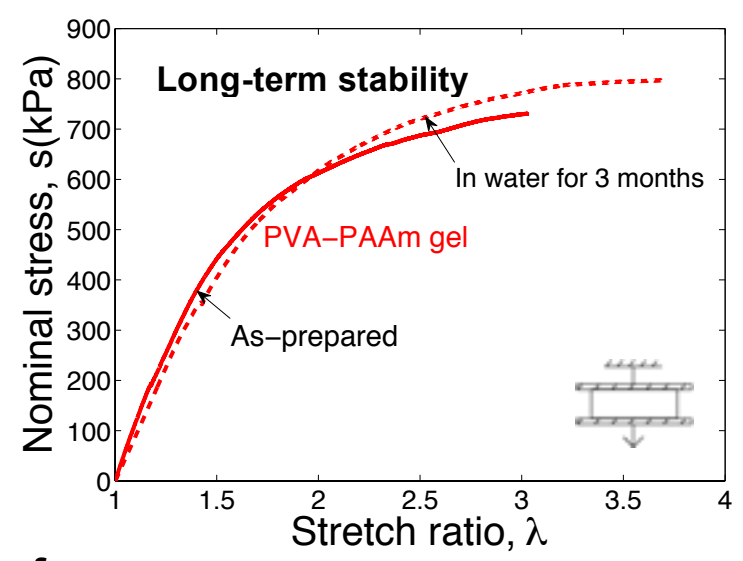

f

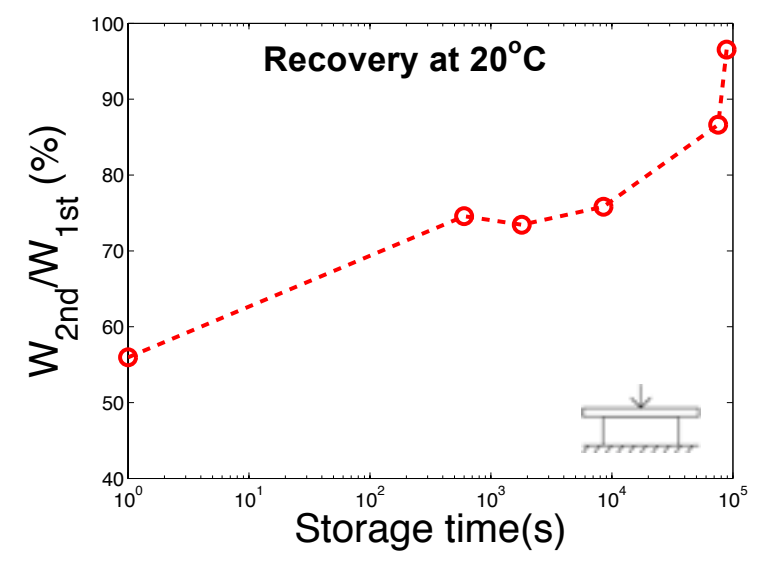

Figure 2. Crystallites as stable and recoverable cross-links. a, Stress-stretch curves of two types of hydrogels. Each sample is stretched to rupture. The nominal stress is the applied force divided by the cross-sectional area of the undeform sample. The stretch ratio is the length of deformed sample divided by the initial length. $\mathbf{b}$, Loading-unloading curves of both hydrogels. 
Each sample is stretched to a stretch ratio of 2.5. c, Effect of the saline solution. The loadingunloading curve of the hydrogels stored in a saline solution $(9 \mathrm{~g} / \mathrm{L})$ overnight (dashed line) is compared with that of the as-prepared hydrogels (solid line). d, Long-term stability of the hydrogels in water. The hydrogels are stored in distilled water for a long period ( 3 months). e, Recovery of samples stored at room temperature for different durations, as labeled. The samples are compressed to a stretch ratio of 0.2 (compressive strain $80 \%$ ). f, Recovery ratio, i.e., work of the second loading, $\mathrm{W}_{2 \text { nd }}$, divided by that of the first loading, $\mathrm{W}_{1 \mathrm{st}}$. 
a
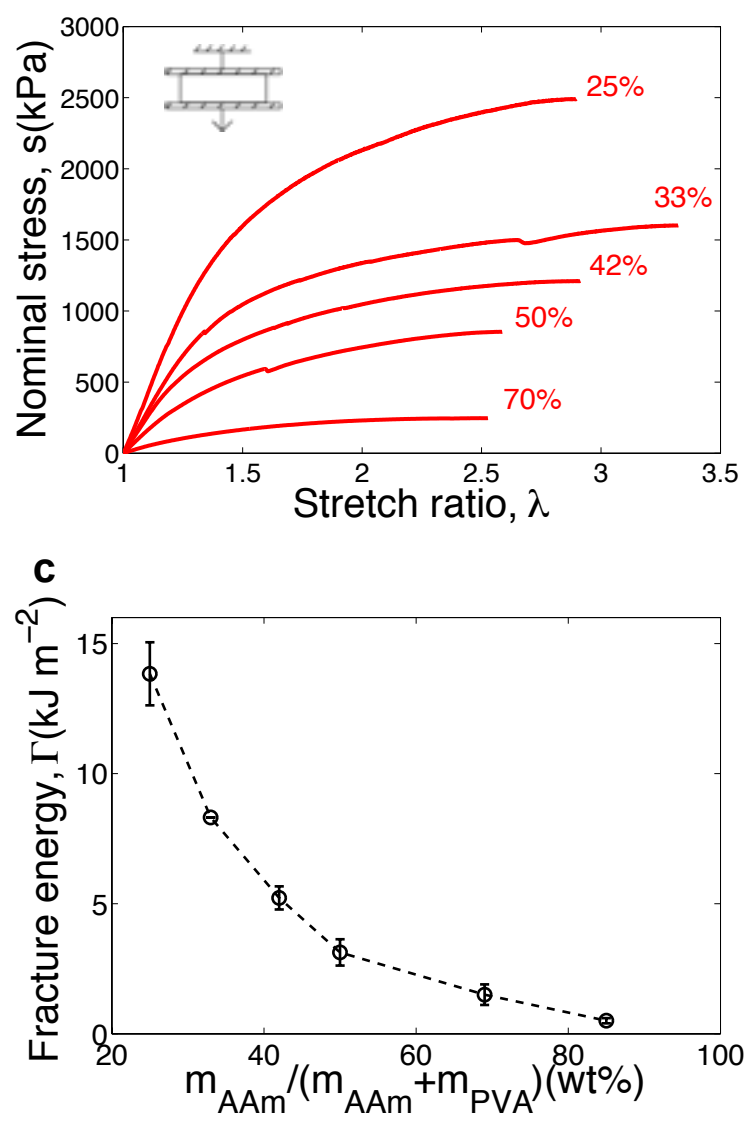

b
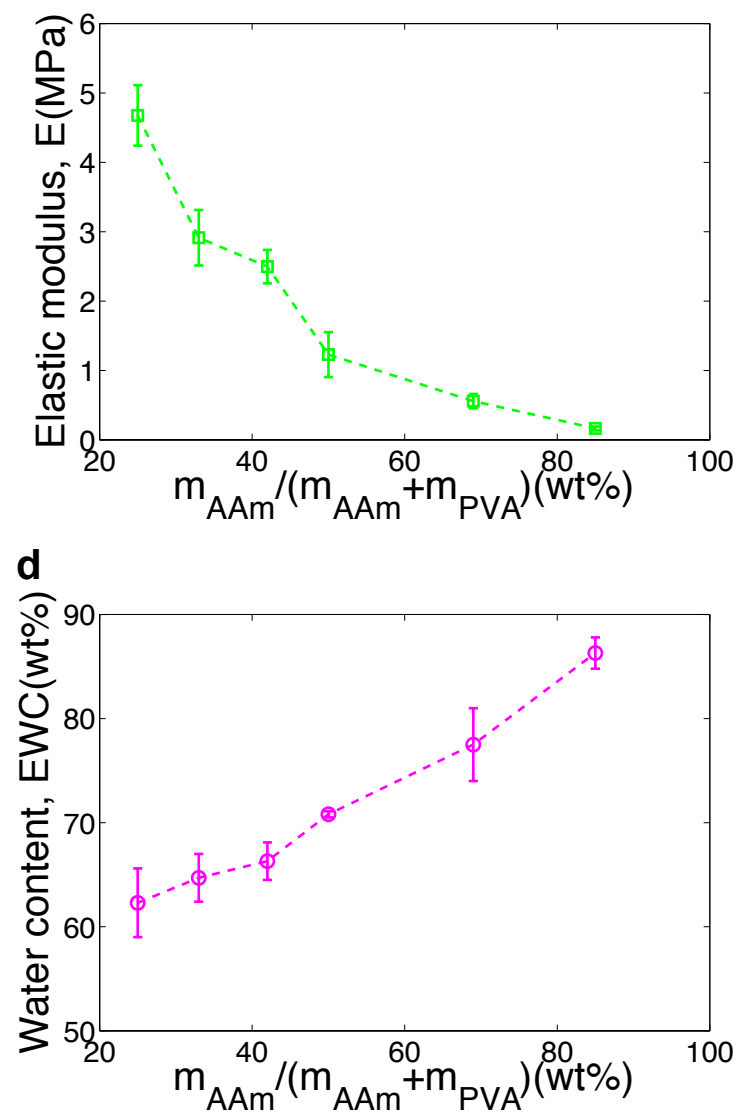

Figure 3. Composition greatly affects the properties of hybrid hydrogels. a, Stress-stretch curves of hybrid hydrogels of various weight percentages of acrylamide to (acrylamide plus polyvinyl alcohol), as labeled. b, Elastic modulus, E, derived from the initial slope of the stressstretch curves. c, Fracture energy, $\Gamma$, as a function of the acrylamide content. d, Water contents of the hydrogels fully swollen in distilled water, $E W C$, plotted against the acrylamide content. 

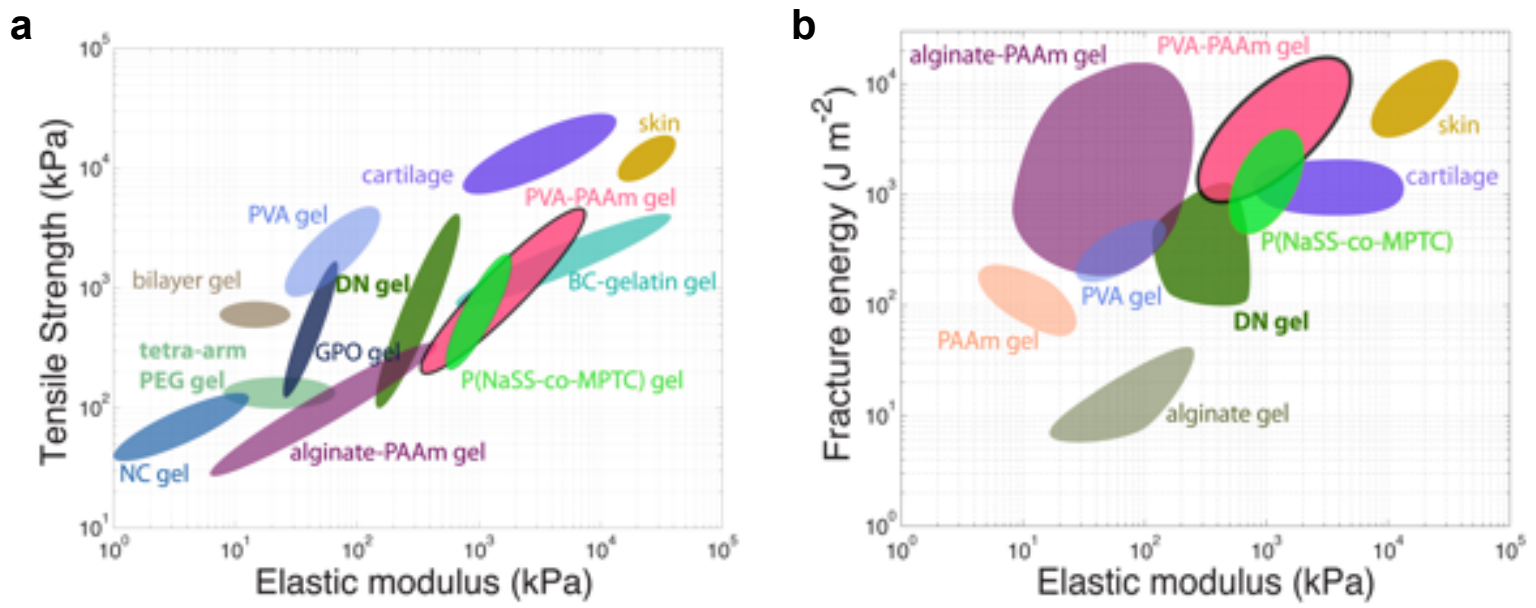

Figure 4. Material property charts for various soft materials. a, Tensile strength versus elastic modulus. b, Fracture energy versus elastic modulus. Materials include the gel prepared in this work (PVA-PAAm), polyvinyl alcohol gel (PVA), ${ }^{22}$ double network gel (DN) ${ }^{16}$ alginatepolyacrylamide $\quad$ gel, ${ }^{3} \quad$ sodium $\quad$-styrenesulphonate-co-3-(methaacryloylamino)propyltrimethylammonium gel (NaSS-co-MPTC) ${ }^{2}$ polyacrylamide gel (PAAm) ${ }^{3}$ alginate gel, ${ }^{3}$ nanocomposite gel (NC), ${ }^{4}$ tetra-arm polyethylene glycol gel (tetra-arm PEG), ${ }^{7}$ hydrophobic bilayer gel, ${ }^{33}$ graphene peroxide gel (GPO) ${ }^{34}$ bacterial cellulose-gelatin gel (BC-gelatin) along with cartilage and skin. ${ }^{30,35}$ 